\title{
The sensitivity analysis of hypergame equilibrium
}

\author{
Zhongfu Qin ${ }^{1, a} \quad$ Xianrong Wei ${ }^{1, b} \quad$ Jingping Li ${ }^{1, \mathrm{c}}$ \\ ${ }^{1}$ College of Civil Engineering and Architecture Zhejiang University,Hangzhou,China \\ aqinzhongfu@zju.edu.cn,'956331238@qq.com, ${ }^{\text {c} 1032484261 @ q q . c o m ~}$
}

Keywords: Hypergame; Equilibrium; AHP; Sensitivity analysis

\begin{abstract}
Hypergame is that people calculate the equilibrium solution of the conflict by judging other players' preference vector according to their own subjective opinion. But in the case of hypergame, to obtain a correct opponent players' preference information is very important. In this paper, AHP and sensitivity analysis are used to solve this problem. Turn a problem into multiple conflict analysis problems, then get each equilibrium of the conflict analysis. In this process the player's cognition to other players can be more accurate. The player can get more stronger stability of equilibrium.
\end{abstract}

\section{Introduction}

Strategy selection of conflict analysis is based on that the information is transparent to each other, and each player is rational and objective. However, the real conflict is not always in the case, information distortion and players error judgment are frequent. For this kind of situation, hypergame technology becomes an effective decision analysis technology. Hypergame is the conflict that one or more players didn't realize the nature of the conflict. It is put forward in the $1970 \mathrm{~s}$ by the western scholars Bennett (1977) [1]. Suppose the method: Due to that the player has a wrong order of other players' preferences or misunderstand others' available strategy, etc., he has a deviation on decision situation [2].

Under the hypergame, getting the proper opponent players preference information is very important. Song Y, (2008) [3] develops an iterative algorithm to integrate the comprehensive results of rival preference information. Because it's difficulty in the process of hypergame to percept the players' preference perception quantitatively. Song Y, [4] ( 2009) presents equilibrium results obtained method which have a fuzzy strategies and preference cognitive. For a certain credibility of decision maker, determine the new strategy set and preference recognition results according to the fuzzy cut and the distance of fuzzy number. In order to reduce the extent of the game in misunderstanding, Gharesifard B, ms Cortes j. [5] (2010) think people can obtain experience by observing the behavior of other players to modify their perspective of other players' payoff function (preferences), the authors introduce a misunderstanding function to measure the differences between player's view and other players' real payoff function.

In order to discuss the stability of hypergame, Sasaki y. [7] (2008), defines a new solution concept that we call the Nash hyper equilibrium stability. This concept solves the stability problem that after each hypergame, misunderstanding retain in the game. Moreover, Gharesifard B, Cortes j.[6](2011), designed a higher-order cognitive update algorithm which allow players continue to update their perception, assuming that players are rational and have perfect observation to the result of the game, 
and use this information to update their preference perception of rivals, so any repeated hypergame will converge to an equilibrium.

This paper, based on the above scholars' research, firstly, finds out the influence factors of hypergame, then conducts sensitivity analysis on the influencing factors. Thus, it builds multiple conflict analysis models, and analyzes the change and stability of the equilibrium solution. Part 2 introduces the hypergame equilibrium model, and Part 3 introduces the sensitivity analysis method of hypergame.

\section{Equilibrium model of Hypergame}

In the stability analysis, hypergame model can be converted into several simple conflict analysis model [8]. In this paper, we analysis the problem from the perspective of the player I. If I's preferences is stable, player J's preferences which player I think is also stable, then, I's equilibrium solution in the game is indeed the equilibrium can solve the conflict which player I think[9.10].

As shown in Table1, Player I thinks Player J has $\mathrm{m}$ preference vectors, Player I's sensitivity analysis is $V_{i j}^{m}$ the corresponding game is that Player I cognitives game ${ }^{G_{i}^{m}}$. This article does not consider Players J mistake cognition, namely Player J have no misunderstanding to Player I. Player J can correctly perceive Player I, so every time Player J's game is only ${ }^{G_{j}}$. Considering the whole hypergame, ( $G_{i}^{m}, G_{j}$ ) will get the ultimate equilibrium solution.

Table 1 Two-person game in hypergame

\begin{tabular}{|c|c|c|c|c|}
\hline Players' cognition & \multicolumn{3}{|c|}{ Players' perception of game } \\
\hline $\begin{array}{c}\text { Player I cognizes } \\
\text { preference of Vector I }\end{array}$ & $V_{i i}$ & $V_{i i}$ & $\ldots \ldots .$. & $V_{i i}$ \\
\hline $\begin{array}{c}\text { Player I cognizes } \\
\text { preference of Vector J }\end{array}$ & $V_{i j}^{1}$ & $V_{i j}^{2}$ & $\ldots .$. & $V_{i j}^{m}$ \\
\hline $\begin{array}{c}\text { The game Player I } \\
\text { cognizes }\end{array}$ & $G_{i}^{1}$ & $G_{i}^{2}$ & $\ldots .$. & $G_{i}^{m}$ \\
\hline $\begin{array}{c}\text { Player J cognizes } \\
\text { preference of Vector I }\end{array}$ & $V_{j i}$ & $V_{j i}$ & $\ldots . .$. & $V_{j i}$ \\
\hline $\begin{array}{c}\text { Player J cognizes } \\
\text { preference of Vector J }\end{array}$ & $V_{j j}$ & $V_{j j}$ & $\ldots . .$. & $V_{j j}$ \\
\hline $\begin{array}{c}\text { The game Player J } \\
\text { cognizes }\end{array}$ & $G_{j}$ & $G_{j}$ & $\ldots .$. & $G_{j}$ \\
\hline Total game & $\left(G_{i}^{1}, G_{j}\right)$ & $\left(G_{i}^{2}, G_{j}\right)$ & & $\left(G_{i}^{m}, G_{j}\right)$ \\
\hline
\end{tabular}




\section{Sensitivity Analysis of equilibrium solution for hypergame}

The order of the initial preference vector of player $\mathbf{i}$ to player $\mathbf{j}$ by chromatography analysis. Assuming in a hypergame Player I thinks that there are $\mathrm{m}$ factors(Evaluation standards) influence its judgment about Player J's preference vector sequence, as shown in Fig. 1. And each evaluation standard has a certain weight value, respectively, f1, f2, ...,fm, $\sum_{m=1}^{m} f_{m}=1$. Using analytic hierarchy process(AHP) method to calculate the weight, then the several evaluation criteria for pairwise comparison, compared to the values known for dominance $(9 、 7 、 5 、 3 、 1 、 1 / 3,1 / 5,1 / 7,1 / 9)$, according to the advantage, calculate weight values. Then all the outcomes (assuming has $\mathrm{K}$ outcomes) are of pairwise comparison under the each standard influence. With the method of AHP, calculate its weight value under each evaluation criterion.

Finally, weight of influence factors and outcome scores are weighted summation, find out the final score ${ }^{\alpha_{i k}}$ of each end of the cognition of Player I

$$
\alpha_{k}=\sum_{m=1}^{m} C_{m k} \bullet f_{m}
$$

Finally, sort the preference vector, according to the calculated value from big to small are arranged, the initial preference vector $V_{i j}^{0}$ of player i to player $\mathrm{j}$.

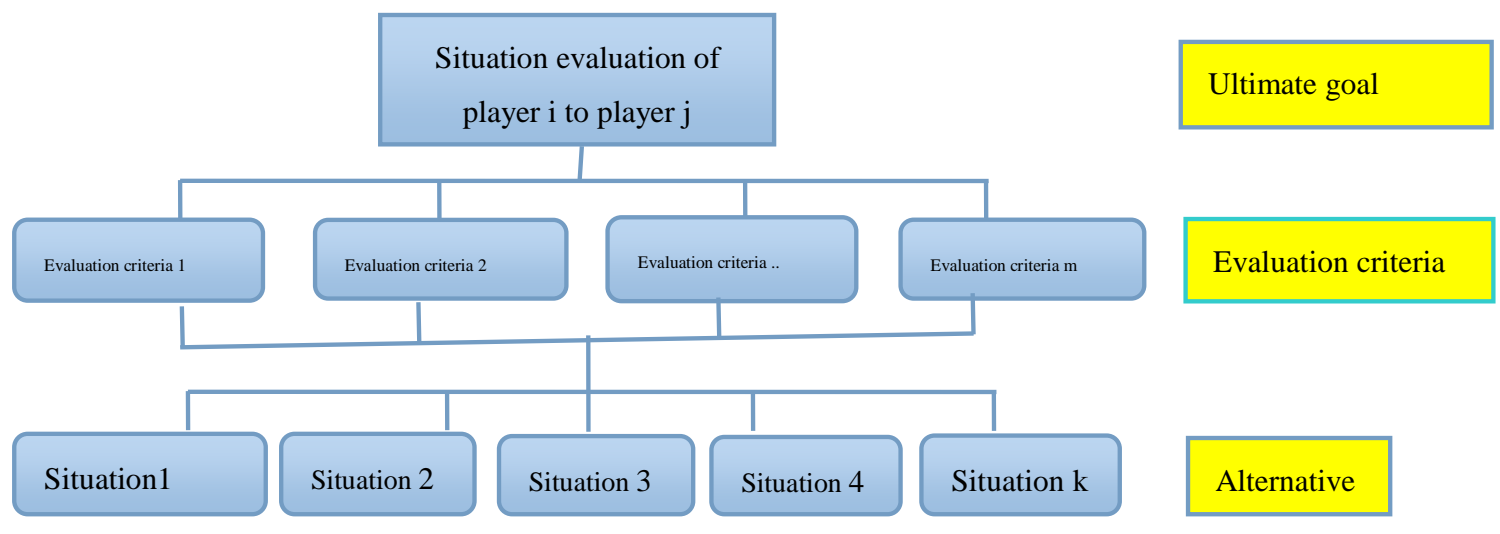

Fig. 1 Hierarchical analysis diagram

Sensitivity analysis on the cognitive preference vector of Player I to Player J. The dominance change of $m$ evaluation standards for pairwise comparison, hange at a close of each value for a time, were changed to $\mathrm{n}$ times, $\mathrm{n}=1,2, \ldots, \mathrm{n} \mathrm{n}=2 \mathrm{~m}$;

Calculate the weight values $f_{m n}$ of the evaluation criteria after each change.

Getting the final score of each outcome of the player i $\alpha_{i k}: \alpha_{k n}=\sum_{m=1}^{m} C_{m k} \bullet f_{m n}$;

To sort $\alpha_{k n}$, get the preference vector $V_{i j}^{n}=\left\{\alpha_{k n}\right\}$ ( ${ }^{\alpha k n}$ sorted from high to low);

Draw preference vector sensitivity analysis chart, statistics preference vectors' type and number; 
Find equilibrium solution from $\mathrm{n}$ kinds of preference vectors. Analyze the equilibrium solution from Player I and equilibrium solution of total problem .

\section{Discussion and summary}

Calculate the equilibrium solution under each preference vector, and statistic the types and the number of equilibrium solution, and compare them with the initial equilibrium of Player I.

If consistent, suggesting that Player I's prediction of the equilibrium is relatively stable, and may be easy to appear in the real world. The player I can stick to its current judgment on other players' preference vector. To note here, it can stick to this kind of judgment, which doesn't mean it can think of this kind of judgment is right, because of what it knows, even if the current preference vector judgment of other players and real preference vector are difference, it also does not affect its rightly prediction of the equilibrium.

If not consistent, Player I should change the preferences vector views to J. Should Player I choose the equilibrium solution to appeare the most frequently? The frequency highest equilibrium is not necessarily in the most satisfactory solution, it is just the most stable solution. This solution is likely to let one player feel very unsatisfactory. Sometimes even let all the parties feel unhappy. Second, though the equilibrium solution is the most stable solution, the reality of the final outcome of the dispute is not necessary in this solution. For example, Player I thinks Player I has A, B, C, D, E possible preference vectors, but it cannot confirm the true preference vector. If $\mathrm{A}, \mathrm{B}$ and $\mathrm{C}$ preference vectors' equilibrium solution is in situation 1 , under $\mathrm{D}$ and $\mathrm{E}$ preference vector ,the equilibrium solutions are in situation 2 and situation 3, but the real preference vector of player $\mathbf{J}$ in the bureau is D. So this case seeks equilibrium solution is a little complex, whether Player I thinks the initial preference vector and the highest number of preferences vector are probably the real preference vector of Player I in bureau, but it might not be.

How to deal with this situation, the author has not studied in this part. This paper has not discussed in detail. But the author will discuss this question in the future.

\section{References}

[1]N. M. Fraser and K. W. Hipel, Conflict Analysis: Models and Resolutions [D]. Systems Science and Engineering, Oxford, UK: Elsevier,1984.53-73.

[2]j., G. Benntt: Bidders and dispenser: manipulative hypergames in a multinational context. European Journal of Operational Research 4 (1980) 293-306.

[3]Xiu Y, Song Y, Zhang J. An Outcome preference Information Aggregation Model and Its Algorithm in Hypergame Situations[C]//Fuzzy Systems and Knowledge Discovery, 2008. FSKD'08. Fifth International Conference on. IEEE, 2008, 4: 494-497.

[4]Song Y, Li W, Zeng X. $\lambda$-equilibrium of hypergame with fuzzy strategy and preference perceptions[C]//Intelligent Computing and Intelligent Systems, 2009. ICIS 2009. IEEE International Conference on. IEEE, 2009, 2: 641-645.

[5]Gharesifard B, Cortés J. Evolution of the perception about the opponent in hypergames[C]//Decision and Control (CDC), 2010 49th IEEE Conference on. IEEE, 2010: 1076-1081. 
[6]Gharesifard B, Cortés J. Learning of equilibria and misperceptions in hypergames with perfect observations[C]//American Control Conference (ACC), 2011. IEEE, 2011: 4045-4050.

[7]Sasaki Y. preservation of Misperceptions - Stability Analysis of Hypergames[C]//proceedings of the 52nd Annual Meeting of the ISSS-2008, Madison, Wisconsin. 2008, 3(1).

[8] Lu Xiangna, conflict analysis, a new decision-making method [J], journal of management engineering, 1993,125-131.

[9]Yong L B S Y Q. Interactive Integration Model of Hypergames with Fuzzy Strategy and Preference Perceptions [J]. Computer \& Digital Engineering, 2011, 2: 002.

[10]Vane R, Lehner P. Using hypergames to increase planned payoff and reduce risk[J]. Autonomous Agents and Multi-Agent Systems, 2002, 5(3): 365-380. 